International Journal of Distributed and Parallel Systems (IJDPS) Vol.2, No.1, January 2011

\title{
Taxonomy for personalized Notification System for Improving the Quality Service of Mobile Marketplace
}

\author{
Amer Ali Sallam ${ }^{1}$ and Siba K. Udgata ${ }^{2}$ \\ Dept. of Computer \&Information Sciences, A.I Lab, University of Hyderabad, India \\ amer.sallamegmail. com ${ }^{1}$ \\ udgatacseuohyd. ernet. in ${ }^{2}$
}

\begin{abstract}
We propose the notion to define an architecture that realizes interaction and integrated notification between mobile devices users and merchants. This architecture allows user to specify a wide variety of interest through various gateways. It aggregates data from numerous sources and makes different type of interaction possible in single framework. The proposed architecture allows notification between different systems running on different platforms. It also accesses information in different modes from various mobile devices users. Moreover, it brings up the need of publishing and subscribing for information of interest. Furthermore, this system integrated an intelligent recommender system and designed to deliver the information of interest by user with imminent or immediately forthcoming events with applicable recommended notification. This model is suitable for environments that need strong personalization of services available in the mobile market place, where the convenience of data delivering request has fixed dead-line.

This architecture not only helps in getting the items having high degree of interest, but also plays important role in revenue increase, cost reduction and enhancing the ability of electronic finance. More effectively framework has the potential to increase consumer satisfaction, enhance consumer/ company loyalty, and boost overall sales by targeting huge number of customers based on their interest.
\end{abstract}

\section{KEYWORDS}

Profile Management Engine, Notification System, Mobile Merchant Server, Buyer Recommendation Engine, Electronic Finance.

\section{INTRODUCTION}

Mobile Market Place (MMP), the traditional E-Market Place combined with mobile devices and wireless networks, is likely to become a major business model in the near future. MMP not only extends Internet-based E-commerce, but also offers a unique business opportunity with its own features, such as ubiquity, accessibility, portability, etc. Nowadays, MMP becomes prevalent in our daily life and mobile users are able and more willing to do business on their mobile devices more readily. However, they are still facing some difficulties to make decision, browsing, searching and buying an item in MMP environment. Moreover, it can be considered as time consuming and frustrating task. Therefore, using recommendation system along with notification system in MMP domain certainly will help to increase consumer satisfaction, enhance consumer/ company loyalty, and boost overall sales through targeting huge number of customers based on their interest and location. However, the MMP offers a business model of buying and selling millions of goods and services via M-commerce. Choosing among so many options is challenging for user. Increasing choice, however, has also increased the amount of DOI : $10.5121 /$ ijdps.2011.2110 
information that users must process before they are able to select which items to meet their needs. In addition, this model typically involves economic activities, interactions between 2 customers and service providers, and commercial transactions crossing companies. To address this information overload, a notification system integrated with recommended system can tackle these problems and makes it easier and more efficient to search, evaluate, and compare items in the MMP through suggesting items for users that are likely to fit their needs, and to provide users with information to help them decide which items to purchase. The items can be suggested based on the users' profiles and their locations, the form of recommendation include suggestion items to the user, providing personalized item information, summarizing community opinion, and providing community critiques. However, user may visit the recommendation system with different mobile devices. The final hybrid recommendation result must be presented according to the characteristic of user's mobile device. Nowadays, we can find many mobile recommendation algorithms [1], [2] and hybrid recommender approaches [3], [4], [5] suitable for mobile environment

Today the internet technologies continue to develop, popularity of mobile terminals to increase, and the quantity of information on the internet is immense and increasing each day. These qualities make mobile agents useful for the information-rich and communication-rich environment such as M-Commerce. All this much of information-rich becomes difficult to be monitored by users where searching for specific data may cost a lot of time. Using Notification System (NS) can help in this matter through supplying user by the data of interest when events occur or certain conditions are met via a convenient means [6]. The means such as instant messaging, e-mail, cellular phone, or WAP, etc. are controlled by the user. Therefore, demands for mobile services become stronger and more diversified. Mobile technology services need to integrate with computer network services. The integration between information services (e.g. databases), notification services (e.g. E-mail, alarm systems) and telecommunications infrastructure (e.g. GSM, Fax, etc.) is one of the strategic issues to satisfy mobility needs [7].

The introduction to MMP should bring many benefits to consumers, service providers, and telecommunication operators. From the customer point of view, customers can access ondemand, at the point of purchase and obtaining best prices in the MMP. This can happen via mobile application. Although MMP provides a technology to increase merchants' sales, it enhances management efficiency for service providers. As companies exchange item information with customers in time according to their personalized preference and locations. And that can be done via a web page promotion or a mobile alert to increase their willingness to buy an item. Also there is some portion going to telecommunication operators. The more the MMP's services are used by customers through mobile, the more revenue can be achieved by the telecommunication operator. In addition, the operator can also achieve revenue via fees charged to service providers for each MMP transaction. However, there are still quite a lot of issues that have to be addressed and revolved for different players in order to have adequate MMP.

NS is one of the most important components in ubiquitous computing of MMP. The main functions of this system are: matching buyers and sellers, facilitating the exchange of information, goods, services, and payments associated with market transactions. Therefore, this system needs to continually collect and integrate data distributed among huge set of users, sites and applications. As a result, it would help users in gathering relevant information of interest and enabling users to decide in timely manner. In addition, within this system, data would be exchangeable through a very large number of devices and heterogeneous type of information 
resources [8]. This may lead to server resource contention, network overload and congestion. Therefore, the system has to select appropriate methods of data delivery.

Moreover, this architecture speaks beyond the traditional way of notification and tries to address the need of user and service provider satisfaction in MMP domain. And it is unique in that it provides a feasible architecture that takes advantage of today's technologies, including the Internet and WAP, to transmit highly important information. In addition, With regard to recommendations and notification alerts, there is no equivalent system that has been addressed 3 so far in MMP domain as a means of urgent missing of user information. At the time of this writing only few traditional news broadcasts and a system to transmit wireless text messages to cell phones have been implemented but they have not pay attention to the user and service provider interest and their location as this architecture do.

The aim of this paper is to present an innovative for increasing the notification efficiency of mobile along with recommender system to meet the need of mobile users (i.e., customer, service provider) and enhance the usage of MMP. With the innovative approach, personalized recommendation and notification can be provided as a guide for mobile users.

The rest of the paper is organized as follows: section 2 discusses related work, section 3 discusses the system characteristics, and section 4 discusses the architecture of proposed model.

\section{RELATED WORK}

The literature shows that notification systems have been studied and implemented for different projects, protocols, and architectures. i.e., web services based event notification systems [9], CORBA notification service [10], and the publish/subscribe communication paradigm [11]. However, notification mechanisms still need some extending with intelligence, and anticipating changes, as foreseeable location updates.

No doubt that Internet and the wireless supply an actual MMP all over the world without location and time restriction. These distinguished features give superiority to M-commerce to dominate the space of commerce instead of e-commerce. Thus, these features play an important role to boost overall sales through targeting huge number of customers based on their interests and locations.

We believe that NS is one of the most important units in MMP and it provides the necessary information to various units of MMP [12], [13] with respect to their request. Undoubtedly, this unit will enhance the efficiency of mobile market place if it does consider the location and preferences for both user and service provider. Usually, mobiles MMP make available on the Internet electronic catalogs that support lists of items or services, price information and commercial transactions. Depending on that, it will return a list of items or filtered services which meet the requirements specified by the buyer. According to the huge number of agencies i.e., broker, auction, delivery, payment, supplier, buyer, etc. and overloaded information interacted and presented between them, NS is considered the backbone of MMP and important unit.

The proposed architecture of notification service responsible for publishing and advertising goods and services offered by the existing brokers and provide channels to communicate with other MMP components which assist to obtain useful information about location and maps, which can help to point the exact position of the item in the market, and gives information 
International Journal of Distributed and Parallel Systems (IJDPS) Vol.2, No.1, January 2011

about location and city roads. Also the user or merchant based profile could be useful to provide information about user requirement, merchant offers or item specification. This type of service has impact factor in enhancing the finance capability of mobile commerce, increase the revenue, reduce the cost and time consuming while search for particular item.

In addition, there are two advantages of NS. First, effective personalized advertising and efficiently presents targeted advertising to users that are likely to purchase items and services; this thereby dramatically improves sales and businesses and makes more profit. Second, the more personalized the advertising becomes; the more customers eventually regard this service not as an intrusive extra but rather as a helpful necessity.

Moreover, this architecture is important for both customers and services providers to confirm the transaction of payment, and provides necessary information that could handle the delivery process of items to the customers.

Mobile notification services designed for particular services have limited number of models [14]. Extensibility is not supported by the most of these models. Previous research has shown criteria that should satisfy the adaptive mobile notification [15], [16], [17], [18], [19] as:

- Take the mobility of users into account: manage highly model of users' interests (subscription, update, insertion, deletion).

- Manage changes in the underlying network topology that may occur in very dynamic setting like an ad hoc networking.

- Support multiple notification channels and multiple content formats: support heterogeneous notification channel (e-mail, internet protocols, fax, phone, WAP, etc.).

- Support large numbers of subscribers and publishers: continuously collect and integrate data distributed among a large set of users.

- Propagate notifications for thousands of information consumer simultaneously, which managing large amount of content sent to the system.

- Select the appropriate mode of communication: filtering and delivering relevant data to interested users and components in a timely manner with helps of middleware which gives filtered formation for potentially large set of users, gives their continuously changing location, their changing profiles.

- Support security and privacy of information: performing security functions, like subscriber and publisher authentication, secure content distribution, i.e., not all subscribers may be allowed to receive all publications that match their subscriptions.

Here we integrate all of these criteria into our proposed model in addition to some criteria that will be addressed in system architecture section.

The literature shows that mobile notification models are designed to have high user interactivity and low user perceived latency. Real-time dynamic web data such as news headlines, stock tickers, and auction up-dates need to be propagated to the users as soon as possible. However, these applications still suffer from the limitations of the Web's request/response (pull-based) architecture which prevents servers from pushing real-time dynamic web data. Such applications usually use a pull style to obtain the latest updates, where the client actively requests the changes based on a pre-defined interval. It is possible to overcome this limitation by adopting a push style of interaction where the server broadcasts data when a change occurs on the server side. Both options have their own trade-offs [20]. 
International Journal of Distributed and Parallel Systems (IJDPS) Vol.2, No.1, January 2011

Data dissemination involves the delivery of data from one or more sources to a large set of consumer [21]. Many dissemination oriented applications have data access characteristics that differ significantly from the traditional notion of client/server as embodied in navigational web browsing technology [22]. Table 1 illustrates the characteristics of dissemination oriented mechanisms.

\begin{tabular}{|c|c|c|}
\hline \multicolumn{2}{|l|}{ Pull } & $\begin{array}{l}\text { Request data when it is required, when request is received at server, the } \\
\text { server locates the information of interest and sends it into the client. }\end{array}$ \\
\hline \multicolumn{2}{|c|}{ Push } & $\begin{array}{l}\text { Data delivery involves sending information into a client population in } \\
\text { advance of any specific request. }\end{array}$ \\
\hline \multicolumn{2}{|c|}{ Periodic } & $\begin{array}{l}\text { Is performed according to some pre-arranged schedule, this schedule may } \\
\text { be fixed or may be generated with some degree of randomness. }\end{array}$ \\
\hline \multicolumn{2}{|c|}{ Aperiodic } & $\begin{array}{l}\text { Is event driven while data request for pull according to any user action, or } \\
\text { transmission for push regarding to data update by server. }\end{array}$ \\
\hline \multicolumn{2}{|c|}{ Unicast } & $\begin{array}{l}\text { Data items are sent from a data source (e.g., single server) to another } \\
\text { machine. }\end{array}$ \\
\hline \multirow[b]{2}{*}{$1-\mathrm{N}$} & Multicast & Data sent to specific subset of clients (recipients is known). \\
\hline & Broadcast & $\begin{array}{l}\text { Send information over a medium on unidentified and unbounded set of } \\
\text { client can listen. }\end{array}$ \\
\hline
\end{tabular}

Table 1: Data delivery mechanisms characteristics

In mobile marketplace notification system, the Mobile Merchant Server (MMS) has the following attributes: there is a huge population of users who want to access the data. There is a tremendous degree of overlap among the interests of the user population. Users who follow the event closely are only interested in new data and changes to the existing data. Therefore, each user sends a request to the server, the large audience for a popular event can generate huge load at servers, resulting in long delays and server crashes. In addition, the use of unicast data delivery likewise causes problems in opposite direction (from server-to-client) with unicast the server is required to respond individually to each request. By considering these characteristics, it becomes clear that the -request/ response- unicast method of data delivery may alone lead to undesirable results. As well as, Scalability considered as elaborate problem in Mobile Notification System (MNS), which can be occurred as a result of mismatch between the data access characteristics of the application and the technology. Furthermore, the mobile devices nature still suffers from the limitation in keyboard and display and the ability of processing. Therefore, MNS needs to use appropriate mechanism of data delivery to enhance the performance of such system. These limitations force researchers to pay special attention to build a flexible system. Notwithstanding, using data push delivery, the transmission of data to user is initiated without requesting the users to explicitly request it, users do not have to poll servers for new and update, the number of user requests that must be handled by server can be reduced dramatically. However, the battle of data push standards is well underway, and the changing from data-pull to data-push does not solve all the problems, and there is no one data delivery that can provide adequate support [17] for the wide variety of data dissemination. Our proposed model supports all delivery mechanisms that have been discussed in table-1. 
International Journal of Distributed and Parallel Systems (IJDPS) Vol.2, No.1, January 2011

\section{SYSTEM CHARACTERISTICS}

The NS is a telecommunication method of delivering a message to a set of recipients. For example, the NS can send an e-mail or notify by another gateway when a new topic has been added to the MMP or when a desired good or service is made available. Moreover, this architecture is important for both customers and services providers to confirm the transaction of payment, and provides the necessary information that could handle the delivery process of items to the customers.

The complexity of the NS is often dependent on the types of messages that must be sent. An e-mail noting when a page has been inserted into MMS is adequate for such a straightforward task. However, the proposed model has the following attributes:

- The ability of notifying individuals' recipients in real-time interaction, or when the events take place.

- Supporting the scheduling and fail-over scenarios.

- The NS can be controlled by mobile user to permit or escalate (spam) the unwanted notification.

- Support customer reviews and item's features and accept them as a feedback to determine what tasks the system should perform.

- Considering users' vacation times, weekends, holidays, so on and so forth, to prevent notifying people when they are not available, otherwise system can notifying them about their interest according to their profiles and locations.

- The ability of distribute the information and ensure the recommended notification has been delivered across the interested gateway by the user, otherwise it should be sent through the second gateway that is preferable by the user in case of failure has occurred to reach the first gateway.

- Supporting the scalability, without changing the model design when there is a need to add new notification service by means of a plug-in approach.

\section{SYSTEM ARCHITECTURE}

\subsection{NSMMP}

Figure 1 depicts the architecture of the Notification System of Mobile market place (NSMMP), as it would be deployed in a mobile market place environment. In such an environment, end users (i.e., customer or service provider) can access the NS and register for their events of interest and obtain the expected content when match has occurred. The architecture of NSMMP system consists of two main components: Customer's Mobile Devices and Mobile Merchant Server, in addition to the interfaces among these components. 
International Journal of Distributed and Parallel Systems (IJDPS) Vol.2, No.1, January 2011

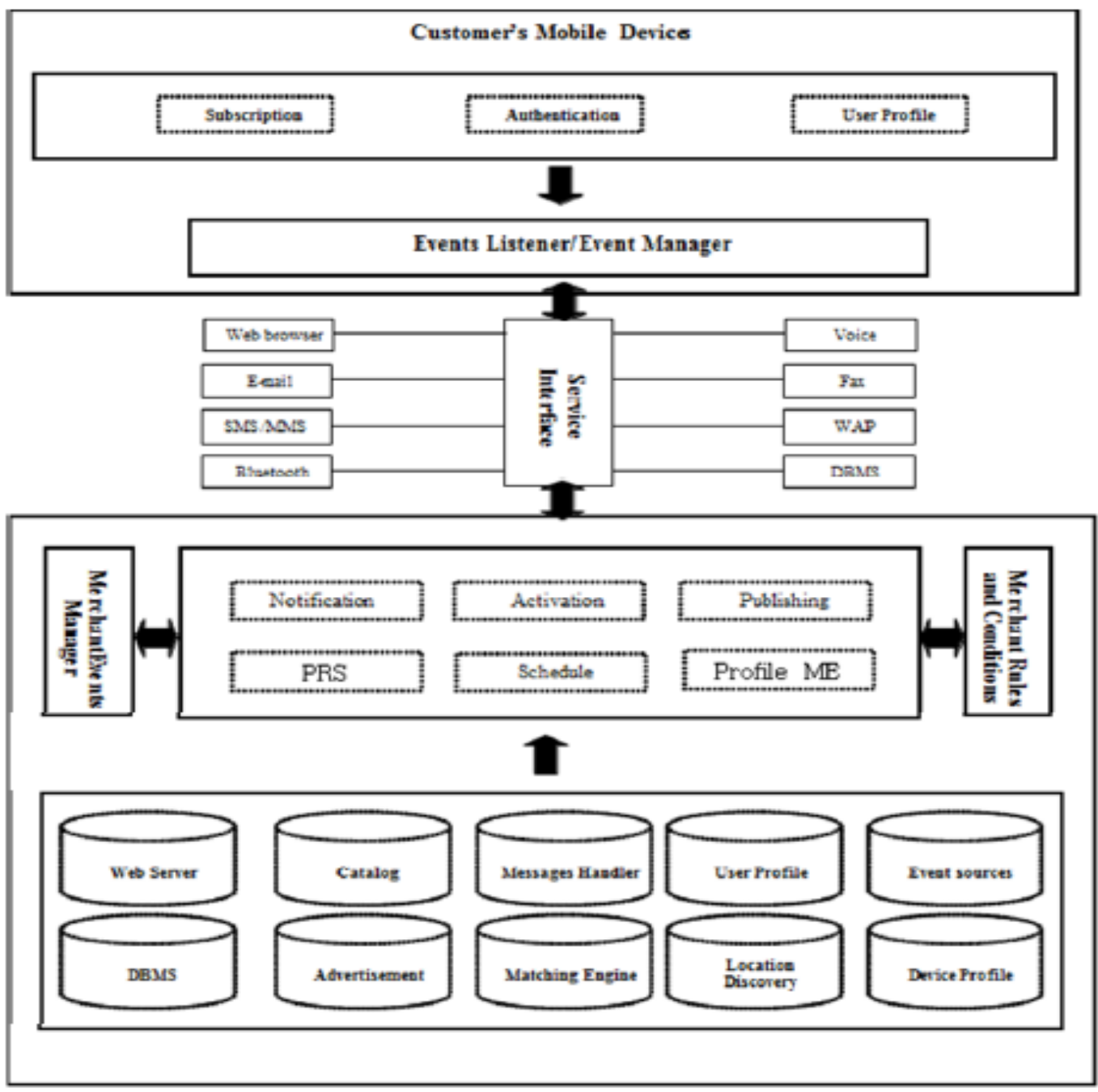

Figure 1: The architecture of the notification system of mobile market place (NSMMP)

\subsubsection{Customer's Mobile Devices:}

The Customer's Mobile Device (CMD) consists of subscription unit, user profile and authentication unit.

\section{The subscription:}

Mobile Marketplace Subscriber (MMPS) is a mobile user that subscribes to events of interest, and the action of this user can be performed at the client's site or at the broker's site. This unit is responsible for performing the subscription activities and making the user able to subscribe for the information of interest or action supplied by MMS or service provider. The subscriber lets the system know through which channel that they like to be informed whenever certain events occur based on location or other conditions. 
International Journal of Distributed and Parallel Systems (IJDPS) Vol.2, No.1, January 2011

\section{User profiles:}

The user profile is defined as data stores that contain information about user, including user preferences, locations, credit card details, authorization and password information and so on.

\section{Authentication:}

Authentication processes should accomplish when the transaction between service provider and mobile user has taken place, and that could be done via an identification process or cryptographic mechanism, though certificates which are trusted, to provide validity of the secrets key which are used for authentication. Those digital certificates used to proof the identity and authentication of each marketplace units.

\subsubsection{Mobile Merchant Server:}

Another main component of NSMMP is the Mobile Merchant Server (MMS). This component has to monitor and handle all users' request and figure out the changeable requirement of users timely. This component consists of:

\section{Notification Unit:}

With the help of this unit, the MMS can notify mobile marketplace subscribers via sending them a text or multimedia message about the available services. Notification is a form of push technology where information is transferred as a result of an event [23] [24]. However, Synchronization notifications, common in mobile networked application designed to provide real-time access to information sources.

\section{Activation Unit:}

If the MMPS is interested in the content of notification, he/she has to reply back to the activation unit to make him/her as a member of this content and allow him/her to receive the data of interest from publication unit in timely manner. In other words, this unit handles the users' activation process and informs the publication unit about each subscriber's interest and his/her preferable gateway that he/she likes to be informed through (e.g., SMS, real-time alert notification, e-mail message, etc.).

\section{Publication Unit:}

While the required of activation processes have been done, and the rules have been met, as well as the events have been occurred, publication unit immediately will publish the events to all customers who have subscribed to that particular event through the most appropriate gateway to the mobile user. In addition, this unit will publish to subscribers all the recommendation events that have been generated by buyer recommendation engine (BRE).

\section{Notification Database:}

Notification system database can be defined as a data store for the heterogonous type of data resources, which is involved by interpreter for the conditions of subscribers' notification, that is associated to the item. This unit can retrieve the condition of information as its availability by their names if the sources of information is a set of files, or generates a logic expression based on table fields in case of the data source was database. The notification mechanism operates as triggers in database, when the event has occurred, the notification action will be fired, if the trigger condition is satisfied. In addition, the database intended for use in heterogeneous 
International Journal of Distributed and Parallel Systems (IJDPS) Vol.2, No.1, January 2011

distributed environments that allow for objects to be shared between different client applications. The goal of the database is to provide highly available and highly reliable storage for items, while supporting safe sharing of these sources by applications working in different platform.

\section{Events and conditions:}

This unit takes into account filtering and controlling the process of those items selected by the subscribers, which are meaningful to their requirement and met with the merchant's rules and conditions. As well as, it's responsible to take input from the user and converting it into standard format.

\section{Profile Management Engine:}

Profile Management Engine (PME) stands for creating, updating, handling and providing the storage of saving relevant information that consists of user preference profile, mobile device profile, user personal information and location.

\section{Buyer Recommendation Engine:}

Buyer Recommendation Engine (BRE) stands for servicing a mobile user community, and providing each user with a list of items which are recently most wanted items by this user based on his profile and location, and identifying target users for a new item. Those goals can be addressed by analyzing profile management engine data and generate the recommendation based on that data. This component is elaborated with more details in next section.

\subsection{RSMMP}

The proposed architecture of recommendation system for mobile market place (RSMMP) consists of several steps as illustrated in Figure 2. There are five components: (1) User agent, (2) Buyer services engine, (3) Profile management engine, and (4) Buyer recommendation engine (5) marketplace. Each component may have several Agents and Mobile Agents. The task of recommendation process is accomplished through the collaboration of all agents. In this work, the main point is to build a recommendation system for mobile market place.

\section{User Agent}

This agent consists of user interface witch is the most top component of the architecture of MMP. This interface provides a GUI that is highly required for supporting mobile user's interaction among the different components of MMP system. This interface has tackled the requirement of providing a convenient way of interacting with the system (the amount of information is specific to the attributes of mobile device), even via mobile phone devices that have many constraints such as small screen with helps of device's profile. This interface also provides necessary services to make the accessibility, and security mechanisms more attractively among the heterogeneousness components of MMP. 
International Journal of Distributed and Parallel Systems (IJDPS) Vol.2, No.1, January 2011

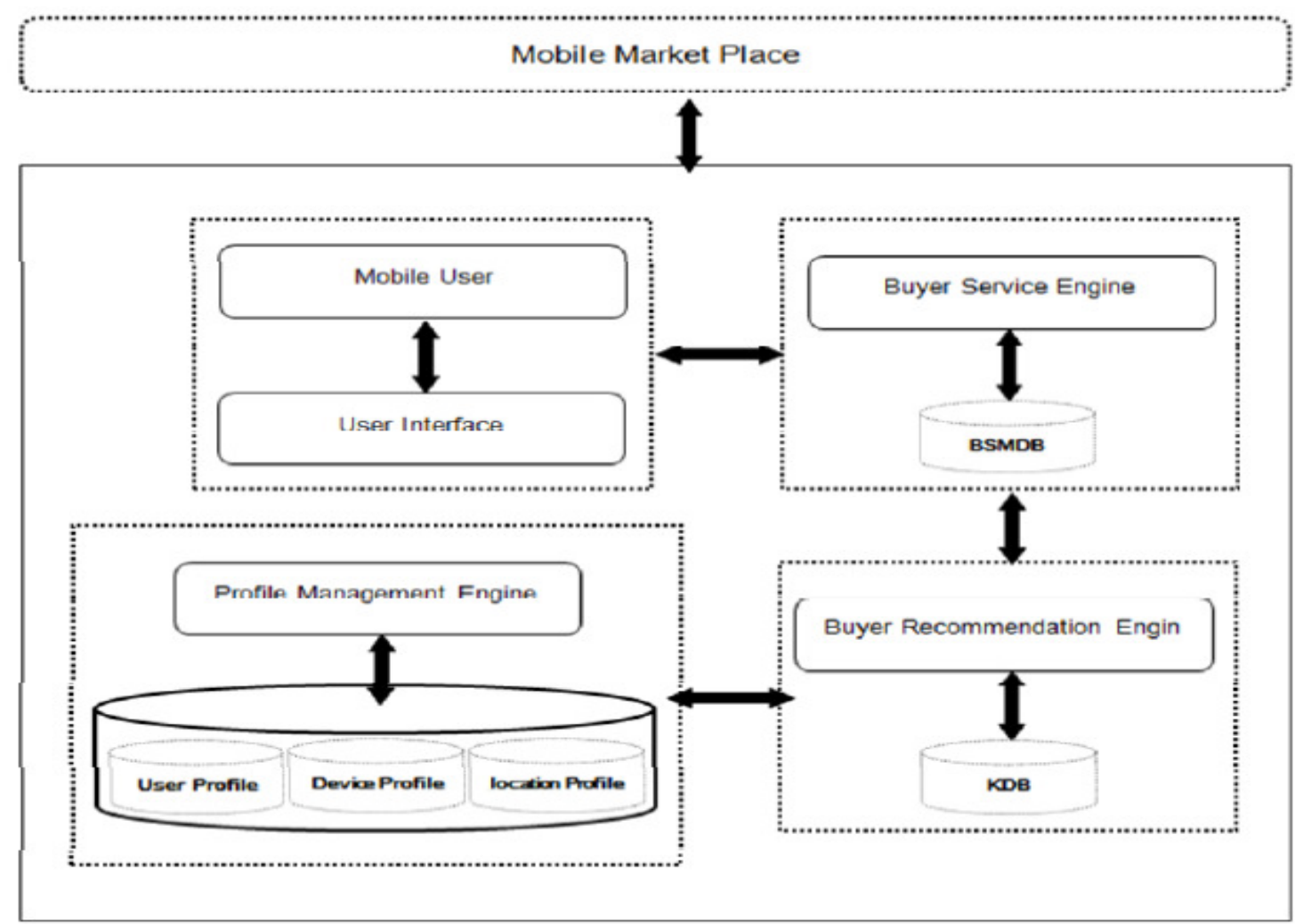

Figure 2: recommender module as a part of notification system (RSMMP)

\section{Buyer Services Engine (BSE)}

The main objective of this component is collecting user behavior data (i.e., navigating pages behavior, purchasing behavior, user rating, feedback behavior for recommendation, etc.). This type of data can be observed and recorded during the processes of interacting user with the heterogeneousness components of MMP. User behavior data is dynamic and do changes as user interacting with system. A user delegates his navigation activities carried out by a mobile device to a mobile merchant agent. It is created at the beginning of an exploration session when a user visits the MMP site and destroyed at the end of the session. This component is necessary to provide accurate input data for different recommendation algorithms.

\section{Profile Management Engine (PME)}

Profile management engine stands for creating, updating, handling and providing the storage of saving relevant information that consists of user preference profile, mobile device profile, user personal information and location. It is always active. Whenever profile management engine receives a request or query from user agent, it generates the relevant profiles information to buyer recommendation engine.

The user profile describes the interest of the user and provides input data to buyer recommendation engine. User profile must involve comprehensive user information that 10 includes static user description data (i.e., gender, age, occupation, etc.) which do not change for time. 
The mobile device profile describes the characteristic of user's mobile device (i.e., screen size, keyboard layout, etc.). It is the guide of content presented to the device as illustrated in figure 3. Many mobile device ontology standards, such as Composite Capabilities/Preferences Profile (CC/PP), User Agent Profile (UAProf) and FIPA (Device Ontology Specification), have been set to describe the capabilities of different mobile devices [25], [26]. Mobile device profile uses standardized format built from CC/PP, UAProf or Device Ontology Specification.

The CC/PP specification defines a high-level structured framework for describing capability of mobile device [27]. CC/PP provides the rules of how to construct a vocabulary that describes capabilities and preferences, but does not specify the actual attribute names and values. The UAProf based on CC/PP, has a base of existing implementations for many devices [28]. The UAProf specification is concerned with capturing classes of device capabilities and preference information that is used only for content formatting purposes.

The sample mobile device profile described in RDF consists of three parts: hardware platform, software platform and WAP characteristics. The hardware platform part describes the hardware information that is associated with user interface format such as screen size, image capable, etc. the software platform part describes the available application style, operation system and Java environment. The WAP characteristics part describes the supported WAP version and script library.

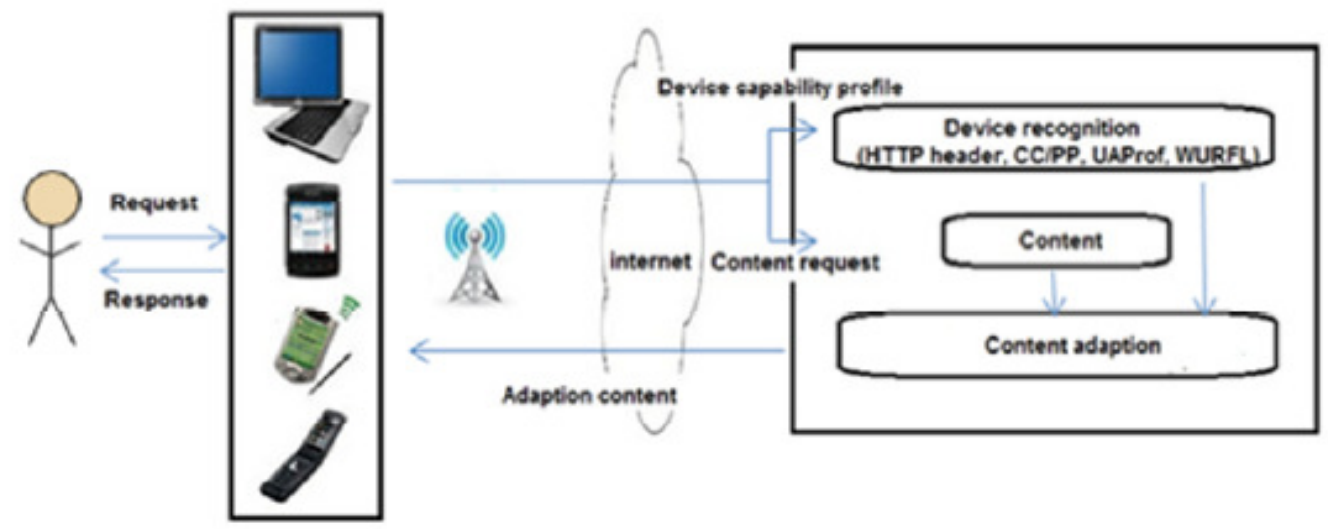

Figure 3: Mobile device profile as a part of NSMMP and RSMMP

\section{Buyer Recommendation Engine (BRE):}

A Buyer Recommendation Engine invokes the weighted of all recommendation algorithms agent. Each recommendation algorithm agent encapsulates a specific recommendation algorithm. It is just to introduce a new agent to encapsulate the new algorithm when a new recommendation algorithm is required. Each agent makes recommendations according to its recommendation mechanism. Then the hybrid algorithm combines and sorts the results and chooses the first $\mathrm{N}$ items as the final recommendation. Finally, BRE transfers the final recommendation list to interface format agent. With the consideration of the steps mentioned above, BRE can stand for servicing a user community, and providing each user with a list of items which are the recently most wanted items by this user and identifying target users for a new item. Those goals can be addressed by analyzing user behavior data. The possibility of each user making another transaction for all types of items is then evaluated in order to identify 
the recently most wanted items. This method provides more accurate results because it is based on each user's entire trading data stored in the database. However, an online retailer may carry 11 several brands for a single item. It is important to carefully examine whether this user is particularly interested in a certain brand. If this user has a high recently repurchase tendency value for a particular item and brand, it's possible to say that there is a high degree of possibility this user will buy the item with the same brand again.

The final recommendation result transfer to the user with helps of interface format agent to format the recommendation according to mobile device profile. Interface format agent reads the mobile device profile and creates user interfaces dynamically according to the characteristic of different devices to present the item and service information.

\section{Marketplace}

A marketplace is the space, actual or metaphorical, in which a market operates. i.e. the "real world" in which items and services are provided and consumed. By another words, marketplace is a place that lets the mobile agents of buyer and seller trade with each other and provides kinds of trading services such as: information query, negotiations, and auctions, etc.

Various factors and services in mobile marketplace play important roles in enhancing the success of current marketing field. The recommender systems are considered as one of the most important tools that make great significance in the marketing activities of M-Commerce.

\section{CONCLUSION AND FUTURE WORK}

Notification services are an essential component of a Mobile Market Place system. As information on the communication technologies become increasingly popular, more and more notification activities are conducted on the mobile market place, and the notification service has played an important role. In this paper, an innovative approach is proposed for developing NS with integrated recommender system to meet the need of mobile users and enhance the performance of MMP skills. We have discussed methods for capturing the notification of mobile market place, and the need of bringing up the necessity of using MNS, which have been considered as a powerful tool in mobile marketplace. While it allows for faster and easier response to market demands and at the same time, consumers can obtain relevant information as well as purchasing goods and services at anytime and anywhere, according to their interest.

The proposed architecture of notification service along with recommender system responsible for publishing and advertising goods and services are highly maintained here. They are offered by the existing service providers and provide channels to communicate with other MMP components. All these help to provide useful information about user location, and help to obtain more details about the items in the market (e.g., availability, price, features, location, and so on). This type of service has also impact factor in enhancing the finance capability of mobile commerce, increases the revenue and reduce the cost.

Moreover, in our proposed architectures for building such system; we are exploring new insights and new adoption behavior in the ubiquitous world of notification system in the mobile marketplace. We believe that the notion of our conceptual model is not yet fully understood by most models of notification system which exists in particular for mobile marketplace. 
International Journal of Distributed and Parallel Systems (IJDPS) Vol.2, No.1, January 2011

In our future work and through this architecture we are going to implement and improve the quality in term of publishing, subscribing to increase the efficiency of notification system along with recommendation system.

\section{REFERENCES}

[1] Gaurav Tewari, Jim Youll and Pattie Maes, (2003) "Personalized location-based rokering using an agent-based intermediary architecture", Decision Support Systems, volume 34, Issue 2, pp. 127137.

[2] Norman M. Sadeh, Enoch Chan and Linh Van, (2002) "An open agent environment for contextaware M-Commerce", 15th Bled Electronic Commerce Conference eReality: Constructing the eEconomy, Bled, Slovenia, June 17-19.

[3] George Lekakos and Petros Caravelas, (2008) "A hybrid approach for movie recommendation", Multimedia Tools and Applications, Volume 36 , Issue 1-2, pp. 55-70.

[4] Nikos Karacapilidis and Lefteris Hatzieleftheriou, (2005) "A hybrid framework for similaritybased recommendations", International Journal of Business Intelligence and Data Mining, Volume 1, Issue 1, pp. 107-121.

[5] Yoshii, K. Goto, M. Komatani, K. Ogata, T. Okuno, H. G, (2008) "An Efficient Hybrid Music Recommender System Using an Incrementally Trainable Probabilistic Generative Model". Audio, Speech, and Language Processing, IEEE Transactions, Volume 16, Issue 2, pp. 435-447.

[6] Nader Mohamed, Jameela Al-Jaroodi, Imad Jawhar, (2008) "A generic notification system for Internet information", IEEE International Conference on In Information Reuse and Integration, 2008. pp. 166171.

[7] Messerschmitt, D.G., (1996) "The convergence of telecommunications and computing: What are implications today?', Proceeding of the IEEE, vol. 84, no. 8, pp. 1167-1186.

[8] Kaasinen, E., (2003) "User needs for location-aware mobile services", Personal and ubiquitous computing, volume 7 , issue 1 .

[9] Huang, Y. and Gannon, D., (2006), "A Comparative Study of Web Services-based Event Notification Specifications," Proc. of ICPP Workshop on Web Services-based Grid Applications (WSGA).

[10] Gore, P., Cytron, R., Schmidth, D., O'Ryan, C., (2001), "Designing and Optimizing a Scalable CORBA Notification Service," Proce. of the 2001 ACM SIGPLAN workshop on Optimization of middleware and distributed systems pp. 196 - 204.

[11] Eugster, P.T., Felber, P., et al., (2003),"The Many Faces of Publish/Subscribe," ACM Computing Surveys, vol. 35 .

[12] Minch, R. P., (2004) "Privacy Issues in Location-Aware Mobile Devices", Proceedings of the 37th annual Hawaii international conference on Systems Sciences.

[13] Bazinette, V., Cohen, N. H., Ebling, M. R., Hunt, G. D. H., Lei, H., Purakayastha, A., Stewart, G., Wong, L., Yeh, D. L. (2001) “An Intelligent Notification System”, IBM Research Report RC 22089.

[14] Engin, B., Ali, M., and Aire, V., D., (2007) "A Comparison of Push and Pull Techniques for Ajax“, TUD-SERG- Report.

[15] Michael, F., Stanley, Z. (1997) “A Framework for Scalable Dissemination Based Systems”, Proc. ACM OOPSLA Conference, Atlanta.

[16] Demet, A., Mehmet, A., Rahul, B., Ugur, C., Michael, F., Jane, W., and Stan Z., (1999) "Research in Data Broadcast and Dissemination” S. Nishio, F. Kishino (Eds.): AMCP’98, LNCS 1554, pp. 194-207. 
International Journal of Distributed and Parallel Systems (IJDPS) Vol.2, No.1, January 2011

[17] Ibach, P., Tamm, G., Horbank M. (2005) "Dynamic Value Webs in Mobile Environments Using Adaptive Location-Based Services," 38th Hawaii International Conference on System Sciences, Hilton Waikoloa Village Island of Hawaii, Big Island.

[18] Adrian H., Imed B., Irek D., (2010) "Notification Service for DVB-H Mobile Broadcast", IEEE Wireless Communications, Vol. 17, no. 2.

[19] Jon C., Jean B., Peter P., George C., and Hani N., (2002) "Channel Islands in a Reflective Ocean: Large Scale Event Distribution in Heterogeneous Networks ", IEEE Communications Magazine, Vol. 40, no. 9, pp. 112-115.

[20] Bazinette, V., Cohen, N. H., Ebling, M. R., Hunt, G. D.H., Lei, H., Purakayastha, A., Stewart, G., Wong, L. and Yeh, D. L., (2001) "An Intelligent Notification System”. IBM Research Report RC 22089.

[21] Afonso, A. P. Silva, M. J., (2004) "Dynamic Information Dissemination to Mobile Users," Mobile Networks and Applications, Volume 9, Issue 5.

[22] Emilio B., Michael S., Saverio N., and Marcus B., (2009) "Automated Real-Time Recommendations for IPTV ", ICC 2009 - IEEE International Conference on Communications, Vol. 32, no. 1, pp. 1444-1449.

[23] Weichang Du and Lei Wang. (2008). "Context-aware application programming for mobile devices". In Proceedings of the (C3S2E '08) conference. ACM, New York, NY, USA, 215-227.

[24] Tilson, D., Lyytinen K., Baxter R., (2004) "A Framework for selecting a Location Based Service (LBS) Strategy and Service Portfolio," 37th Hawaii International Conference on System Sciences, Big Island.

[25] Foundation for Intelligent Physical Agents. FIPA Device Ontology Specification, (2009), available at: http://www.fipa.org/.

[26] Open Mobile Alliance, "Enabler release definition for user agent profile version 2.0", (2009), available at: http://www.openmobilealliance.org/.

[27] Composite Capabilities/Preferences Profile, http://www.w3.org/Mobile/CCPP/.

[28] User Agent Profile, http://www.wapforum.com/what/technical.htm.

\section{Authors}

Amer A. Sallam is a Ph.D. scholar in computer science at the University of Hyderabad, in the Research Scholar Lab under the supervision of Dr. Siba K. Udgata. His research activities focus on the design and implementation of adequate programming abstractions for emerging distributed architectures, such as mobile marketplace with a focus on recommendation and publishing systems.

Siba K. Udgata is an associate professor in computer and information sciences, university of Hyderabad. His main research activities focus on mobile computing, wireless sensor network and Ad hoc network. He was an united nations fellow and currently working as research project for development of WSN application and resource allocation schemes for cognitive radio network.
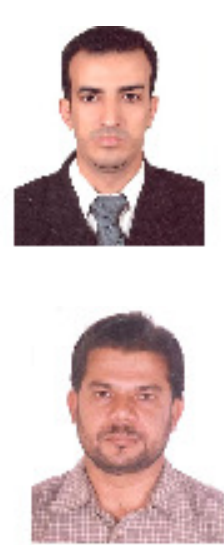